$\xi_{p}$

\title{
Conductivity Studies of Epoxidized PMMA Grafted Natural Rubber Doped Lithium Triflate Gel Polymer Electrolytes
}

\author{
Khuzaimah Nazir ${ }^{*}$, Mohamad Fariz Mohamad Taib ${ }^{2}$, Rosnah Zakaria ${ }^{2,5}$, Muhamad Kamil Yaakob ${ }^{2}$, Oskar \\ Hasdinor Hassan ${ }^{3}$, Muhd Zu Azhan Yahya ${ }^{4}$, Ab Malik Marwan Ali ${ }^{2,5}$, \\ 1Faculty of Applied Sciences, Universiti Teknologi MARA, 26400, Bandar Pusat Jengka, Pahang, Malaysia. \\ 2Faculty of Applied Sciences, Universiti Teknologi MARA, 40450 Shah Alam, Selangor, Malaysia. \\ 3 Faculty of Art and Design, Universiti Teknologi MARA, 40450 Shah Alam, Selangor, Malaysia \\ 4Faculty of Defence Sciences and Technology, Universiti Pertahanan Nasional Malaysia, Kem Sungai Besi, 57000 Kuala Lumpur. \\ 5Institute of Science, Universiti Teknologi MARA, 40450 Shah Alam, Selangor, Malaysia. \\ *Corresponding author E-mail: ammali@salam.uitm.edu.my
}

\begin{abstract}
A gel polymer electrolytes (GPEs) comprising of $62.3 \mathrm{~mol} \%$ of epoxidized-30\% poly(methyl methacrylate) grafted natural rubber (EMG30) as a polymer host, $\mathrm{LiCF}_{3} \mathrm{SO}_{3}$ as a dopant salt and ethylene carbonate (EC) as a plasticizer was prepared by solution-casting technique. The effect of plasticizer on the EMG30- $\mathrm{LiCF}_{3} \mathrm{SO}_{3}$ on the ionic conductivity is explained in terms of the plasticizer loading of the film. The temperature dependence of the conductivity of the polymer films obeys the Vogel-Tamman-Fulcher (VTF) relationship. The ionic transference number is calculated using Wagner's polarization technique shows that the conducting species are predominantly due ions and hence showed the system is an ionic conductor. Surface morphological analysis showed the sample with the highest conductivity exhibited most homogenous in nature.
\end{abstract}

Keywords: conductivity; ethylene carbonate; gel polymer electrolyte; lithium trifluoromethane sulfonate; methyl-grafted natural rubber

\section{Introduction}

Polymer electrolytes are the main concern of researchers due to its ability for new types of electrical power generation and storage systems such as sensors, supercapacitors, solar cell and rechargeable batteries [1-4]. Although liquid electrolyte has a well-known produce high-rate and low-temperature performance, it has the major drawback of leakage. Solid polymer electrolytes (SPEs) prevailingly represent low ionic conductivity at room temperature due to the high crystallinity of the polymers. It is necessary to decrease the glass transition temperature or crystallinity of the polymer and to increase the ionic conductivity by increasing the molecular motion. To overcome these problems, many research efforts have focused on improving the properties of polymer electrolytes viz chemical modification of polymer host, polymer grafting and plasticization with low molecular weight plasticizers [5-9]. Plasticization approach is one of the significant techniques to increase the ionic conductivity and SPEs incorporated with plasticizer are commonly known as gel polymer electrolytes (GPEs) The GPEs, on the other hand, offers a better ionic conductivity than SPEs and possess a superior interfacial electrode-electrolyte contact. However, to produce a good GPE, the selection of a polymer host plays an important role in obtaining the flexibility of electrolyte films. The polymer should be stable, contain good dielectric properties and have the ability to allow ionic dopant salt to dissolve in the polymer matrix for ionic conduction.

Several types of polymer such as poly(methyl methacrylate) (PMMA), poly(vinyl chloride; PVC), poly(vinylidene fluoride; PVDF), poly(ethylene oxide; PEO) and methyl-grafted natural rubber eg: MG30 and MG49 has been studied to see their potential as a polymer-based electrolyte [10-15]. Among them, methylgrafted natural rubber was extensively studied by many researchers due to its good properties, high flexibility and its ability to solvate inorganic salts to form a polymer-salt complex [16-20]. Methyl-grafted natural rubber sheets can be bonded with very good results, both along the edge and on their surface, by using solvent adhesives and gap-filling polymerization adhesives. The excellent physical and chemical properties of MG30 made it of great interest in the polymer electrolyte industry. Yap and coworkers [21] prepared MG30-LiTf-PEG exhibited the highest ambient temperature conductivity in the order of $\sim 10^{-4} \mathrm{~S} \mathrm{~cm}^{-1}$. However, the value of ionic conductivity is insufficient which is need up to $\sim 10^{-3} \mathrm{~S} \mathrm{~cm}^{-1}$ for practical applications. Furthermore, the modified NR based polymer electrolyte is unstable with age. This is due to the presence of carbon-carbon double bond $(\mathrm{C}=\mathrm{C})$ in their polymer chain, which is weak and easily to break-off [2223]. The suitable approach to improve the stability of MG30 can be made via the chemical modification of their structure. Epoxidation of MG30 will reduce the $\mathrm{C}=\mathrm{C}$ in the structure of MG30 and increase the polar group with the formation of the epoxy group (C-O-C). The increase of polarity in EMG30 is expected to increase the ionic conductivity in polymer electrolytes. The characterizations of EMG30 have been discussed earlier in our previous work [24]. MG30 with 9 hours of performic acid reaction could produce EMG30 with $62.3 \mathrm{~mol} \%$ of epoxidation. In this work, the $62.3 \mathrm{~mol} \%$ of EMG30 as a polymer host was doped with various wt.\% of $\mathrm{LiCF}_{3} \mathrm{SO}_{3}$ and $\mathrm{EC}$ plasticizer to form GPEs by using the solution casting technique. These polymer electrolytes were characterized by electrochemical impedance spectroscopy (EIS), differential scanning calorimetry (DSC), electrochem- 
istry analyzer and field-emission scanning electron microscopy (FESEM).

\section{Experimental}

MG30 was obtained from Rubber Research Institute of Malaysia and used as purchased. Lithium trifluoromethane sulfonate $\left(\mathrm{LiCF}_{3} \mathrm{SO}_{3}\right)$ obtained from Aldrich was pre-dried for more than 48 $\mathrm{h}$ under vacuum at $110{ }^{\circ} \mathrm{C}$. The tetrahydrofuran (THF), toluene, methanol and aqueous ammonia, obtained from Aldrich were chemically pure reagents. Both formic acid ( $88 \%$ aqueous solution) and hydrogen peroxide (30\% aqueous solution) were reagent grade and used as received.

The polymer electrolyte was prepared by the solution cast technique with THF as the solvent. $1 \mathrm{~g}$ of the highest epoxidation content of EMG30 (62.3 mol\%) was then dissolved in $40 \mathrm{ml}$ THF for about $48 \mathrm{~h}$. A different stoichiometric ratio of $\mathrm{LiCF}_{3} \mathrm{SO}_{3}$ was dissolved in a different solution of EMG30 (62.3 mol\%) in a closed Scott bottle and continuously stirred for several hours at room temperature until homogenous. The viscous solutions of electrolyte were then cast onto glass Petri dishes and left to dry in a fume hood to evaporate the THF solvent. Freestanding films were further dried under vacuum oven for another $48 \mathrm{~h}$ to remove residual solvent. The composition with the highest conducting sample was used in preparing the plasticized samples. An appropriate amount of EC was added for each section, and the same steps were applied. All resulting samples were stored in a desiccator until further use.

The ionic conductivities of polymer electrolytes were obtained using the $\mathrm{AC}$ complex impedance approached at room temperature and in a temperature range of $100^{\circ} \mathrm{C}-30^{\circ} \mathrm{C}$. The measurements were carried out using HIOKI 3532-50 LCR HiTester over a frequency range of $1-10^{6} \mathrm{~Hz}$. The data were processed using ALL HiTester software. The samples were sandwiched between two stainless steel ion-blocking electrodes with a cross-sectional area of $3.142 \mathrm{~cm}^{2}$. The temperatures of the samples were set in SH-221 Bench-top Type Temperature and Humidity chamber. The total ionic conductivity was calculated using equation (1).

$\sigma=\frac{t}{R_{b} A}$

Whereas $R_{b}$ is the bulk resistance obtained from the intercept on the real impedance axis of the impedance plot, $t$ is the film thickness and $\mathrm{A}$ is the contact area of the thin film. A NETZSCH DSC 300 F3 Differential Scanning Calorimeter (DSC) was used to determine the transition glass temperature of the polymer electrolytes, using aluminium pans. The samples were heated at a rate of $10^{\circ} \mathrm{C}$ min- 1 , over a temperature range of $-80^{\circ} \mathrm{C}$ to $30^{\circ} \mathrm{C}$ under a

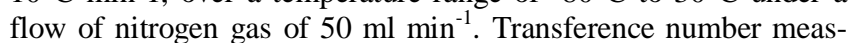
urement was carried out using WBCS 3000 Wonatech Total Electrochemistry Analyzer. The direct current (DC) was monitored as a function of time on the application of a fixed $50 \mathrm{mV}$ DC voltage across the electrolytes with the stainless steel electrodes. The Field-Emission Scanning Electron Microscopy (FESEM) micrograph of EMG30 and with the addition of lithium salts and plasticizer were taken with SUPRA 40VP to examine the morphology and surface structure of the samples at the required magnification at room temperature.

\section{Results and discussions}

The ionic conductivity of polymer electrolytes as a function of $\mathrm{LiCF}_{3} \mathrm{SO}_{3}$ concentration at room temperature $(303 \mathrm{~K})$ is depicted in Fig. 1. It was observed that the ionic conductivity increased rapidly until a maximum of $1.10 \times 10^{-3} \mathrm{~S} \mathrm{~cm}^{-1}$ for $40 \mathrm{wt} \%$ after which the conductivity decreased again. The initial rise in conductivity as the salt concentration increase was due to the increase in the number of the conducting species in the electrolyte. The de- crease in conductivity with higher salt concentration is attributed to ion association or ion aggregation which reduces the number charge carriers [25-26]. However, the addition of plasticizer enhances the conductivity by softening the polymer backbone and increasing free space [25-27].

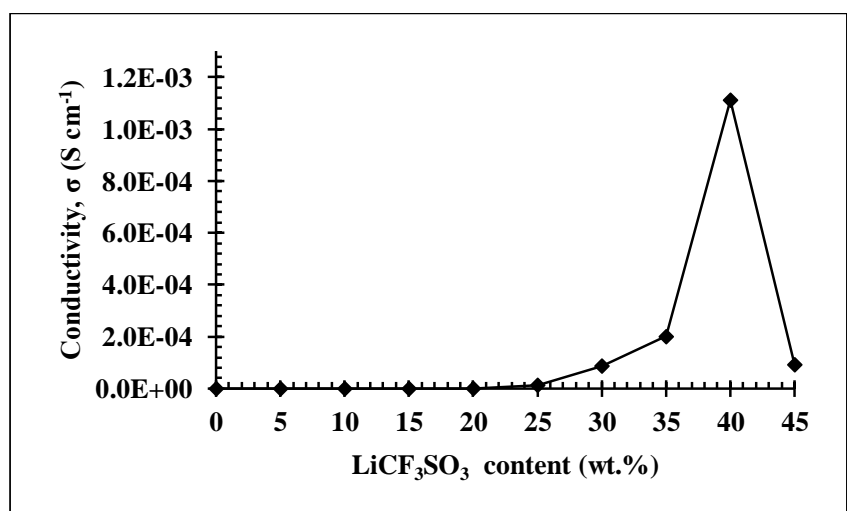

Fig.1: The conductivity plot of the unplasticized EMG30- $\mathrm{LiCF}_{3} \mathrm{SO}_{3}$ system.

The conductivity-temperatures dependence studies for plasticized and unplasticized EMG30 polymer electrolyte was the first plot according to the Arrhenius law in the temperature range of 303 $373 \mathrm{~K}$ (Fig. 2 and Fig. 3). The curvature exists for the $\log \sigma$ vs. 1000/T graph indicates the ionic conductivity does not follow Arrhenius rules. As the temperature increase, the ionic conduction can't be explained by the ionic hopping mechanism as observed in ionic crystals. This behaviour is might be best observed using the Vogel-Tamman-Fulcher (VTF) model which is the ionic movement is dominantly through the viscous rich phase medium.

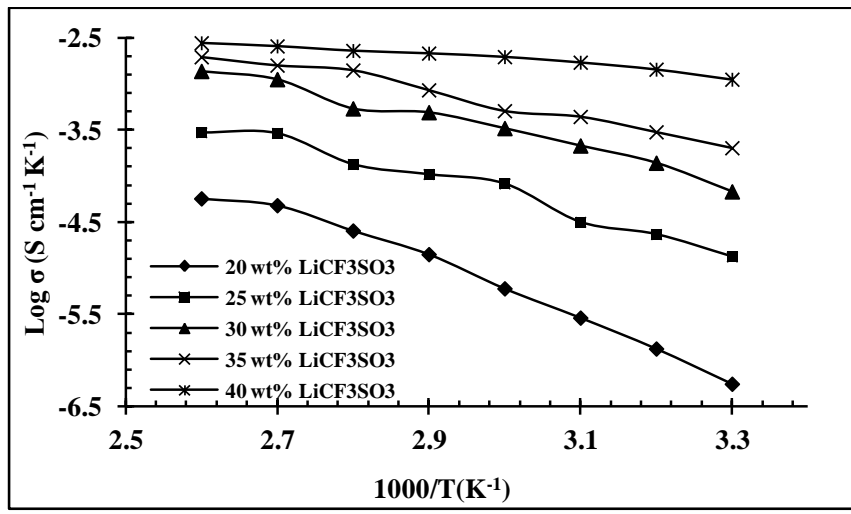

Fig. 2: Arrhenius plot for unplasticized GPE system.

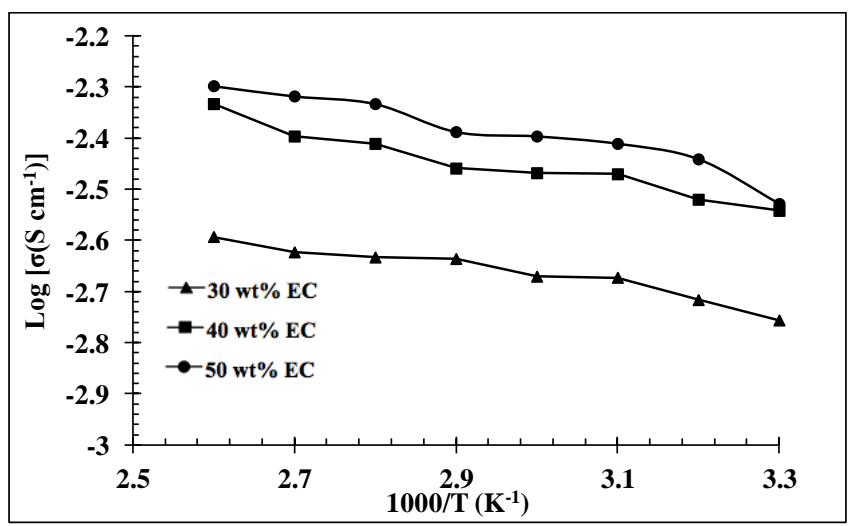

Fig. 3: Arrhenius plot plasticized GPE system.

Fig. 4 and 5 show the VTF plot of $\log \left(\sigma \mathrm{T}^{1 / 2}\right)$ versus $1000 /\left(\mathrm{T}-\mathrm{T}_{\mathrm{o}}\right)$ at room and elevated temperatures for $\mathrm{EMG} 30-\mathrm{LiCF}_{3} \mathrm{SO}_{3}$ and EMG30- $\mathrm{LiCF}_{3} \mathrm{SO}_{3}-\mathrm{EC}$, respectively. The regression value is al- 
most unity for all samples indicating that the conductivity behaviour fitted to the VTF equation (2);

$$
\sigma=\left(\frac{A}{T^{\frac{1}{2}}}\right) \exp \left(\frac{-B}{k_{B}\left(T-T_{\mathrm{o}}\right.}\right)
$$

where $\mathrm{T}$ is the absolute temperature, $\mathrm{A}$ is a constant that is proportional to the number of charge carrier, $\mathrm{B}$ may be considered as a pseudo-activation energy, $\mathrm{k}$ is Boltzmann constant and $\mathrm{T}_{\mathrm{o}}$ is the critical temperature at which the free volume disappear and configurationally entropy becomes zero, usually below 30-50K below $\mathrm{T}_{\mathrm{g}}$. The glass temperature, $\mathrm{T}_{\mathrm{g}}$ can be obtained from Different Scanning Calorimetry (DSC). From the graph, it is observed the increase in conductivity with temperature agree with the previous report based on MG30 [29]. This phenomenon can be explained as the temperature increase the segmental motion of polymer increase. As the temperature increase, the vibrational energy of a segment is sufficient to push against the hydrostatic pressure imposed by its neighbouring atoms and create a small amount of space surrounding its own volume in which vibrational motion can occur agree with the free volume model [30]. Therefore the free volume around the polymer chain causes the migration of ions thus causes to increment of conductivity.

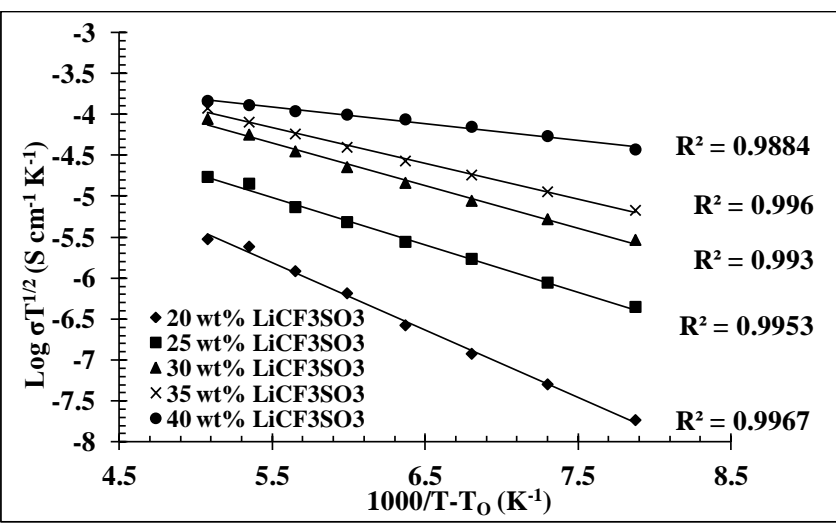

Fig. 4: VTF plot for unplasticized GPE system.

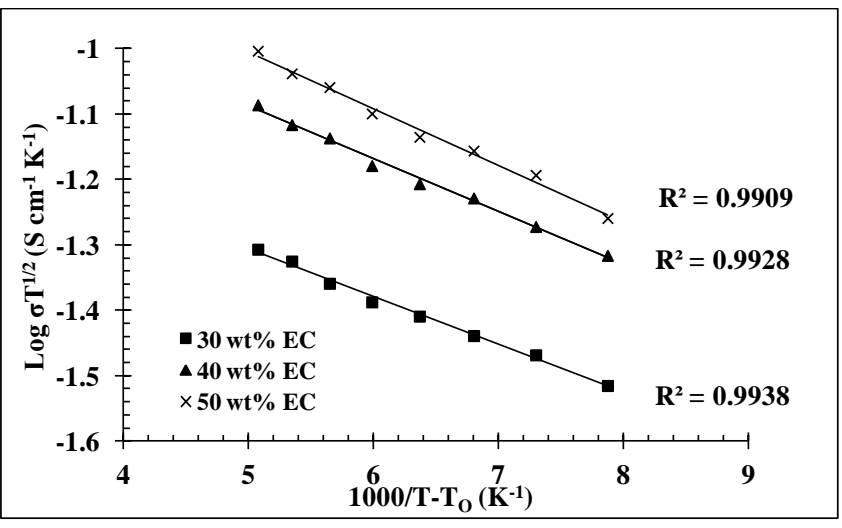

Fig. 5: VTF plot for plasticized GPE system.

The conductivity behaviour of the polymer electrolyte can be explained by dielectric studies. Fig. 6 and 7 shows the dielectric constant as a function of frequency for samples containing different concentrations of $\mathrm{LiCF}_{3} \mathrm{SO}_{3}$ and $\mathrm{EC}$ at room temperature, respectively. In Fig. 5, at the low-frequency region, the dielectric constant rise sharply and decrease toward high frequency. The value of dielectric constant increase with higher salt concentration at a different frequency. The increasing of dielectric constant obtained can be attributed to the increase in a number of mobile ions correlated to ionic polarization. The cations were displaced in the direction of an applied field while anions were displaced in the opposite direction. Clearly, the highest room temperature conductivity of EMG30-salt complexes (40 wt.\% $\mathrm{LiCF}_{3} \mathrm{SO}_{3}$ ) has a high dielectric constant. This signifies the increase of a number of po- larized ions could significantly increase the dielectric. The characteristic of a complex dielectric constant for the plasticized EMG30- $\mathrm{LiCF}_{3} \mathrm{SO}_{3}$ sample (Fig. 7) is similar to that of EMG30$\mathrm{LiCF}_{3} \mathrm{SO}_{3}$ that is dielectric constant increases as plasticizer content increases at the low-frequency region. The strong frequency dispersion of permittivity at low frequency followed by decreasing at the higher frequency. The higher value observed in the complex dielectric constant suggested that the number of polarized ions has increased due to the presence of EC plasticizer and chain flexibility motion of polymer matrix being more favourable.

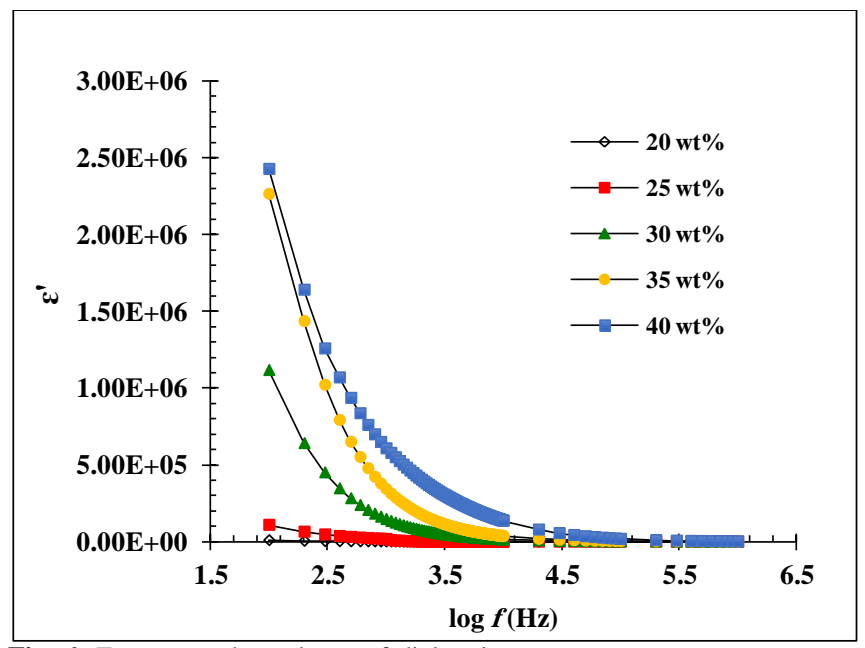

Fig. 6: Frequency dependence of dielectric constant at room temperature for $62.3 \mathrm{~mol} \%$ of EMG30-x wt. $\% \mathrm{LiCF}_{3} \mathrm{SO}_{3}$.

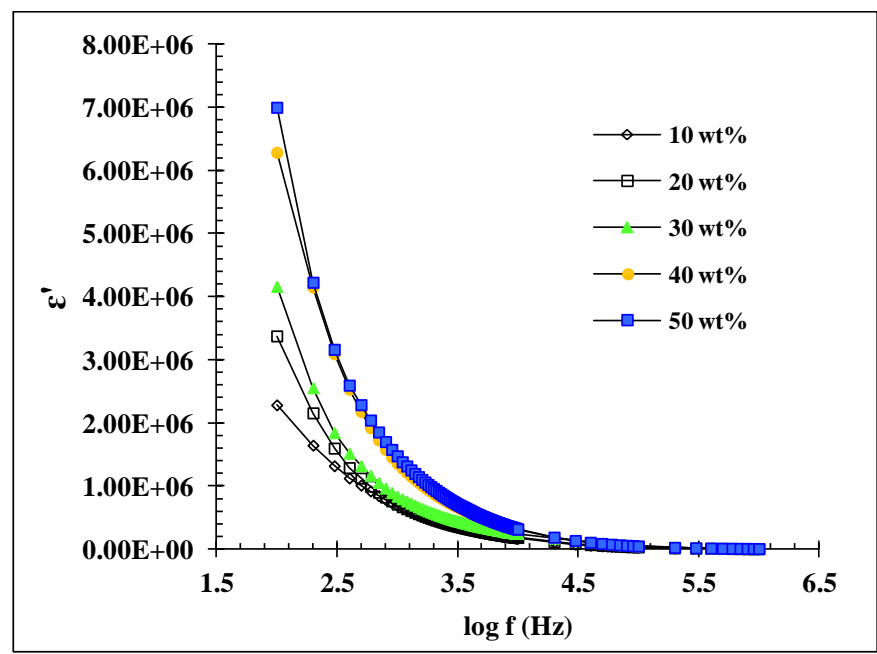

Fig. 7: Frequency dependence of dielectric constant at room temperature for $62.3 \mathrm{~mol} \%$ of $\mathrm{EMG} 30-\mathrm{LiCF}_{3} \mathrm{SO}_{3}-\mathrm{x}$ wt. $\% \mathrm{EC}$.

Fig. 8 and 9 shows the polarization current versus time to evaluate the transference number. The conductivity measurement using blocking electrode is a powerful method for measuring both ionic $\left(t_{i}\right)$ and electronic $\left(t_{e}\right)$ transport. The transference numbers have been evaluated in EMG30- $\mathrm{LiCF}_{3} \mathrm{SO}_{3}$ and EMG30- $\mathrm{LiCF}_{3} \mathrm{SO}_{3}-\mathrm{EC}$ electrolyte systems using Wagner's polarization technique [21]. The transference numbers are calculated from the polarization current versus time plot using the equation (3);

$t_{e}=\frac{i_{f}}{i_{t}} ; t_{i}=1-\frac{i_{f}}{i_{t}}=1-t_{e}$

where $i_{f}$ and $i_{t}$ are the steady state/final and total currents, respectively. The value of the ionic transference number $\left(t_{i}\right)$ is found 0.83 and 0.96 for EMG30- $\mathrm{LiCF}_{3} \mathrm{SO}_{3}$ and $\mathrm{EMG} 30-\mathrm{LiCF}_{3} \mathrm{SO}_{3}-\mathrm{EC}$ electrolyte systems, respectively. This suggests that the charge transport in this polymer electrolyte film is predominantly due to ions and only negligible contribution came from the electron. 


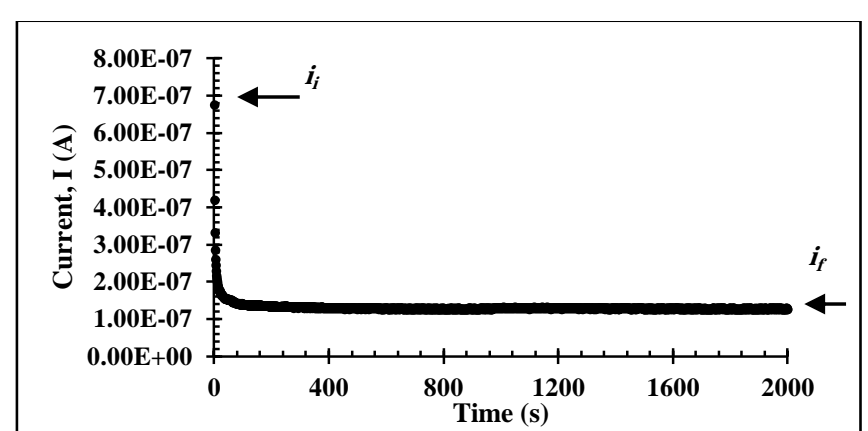

Fig. 8: Polarization Current as a function of time for the highest conductivity of EMG30- $\mathrm{LiCF}_{3} \mathrm{SO}_{3}$.

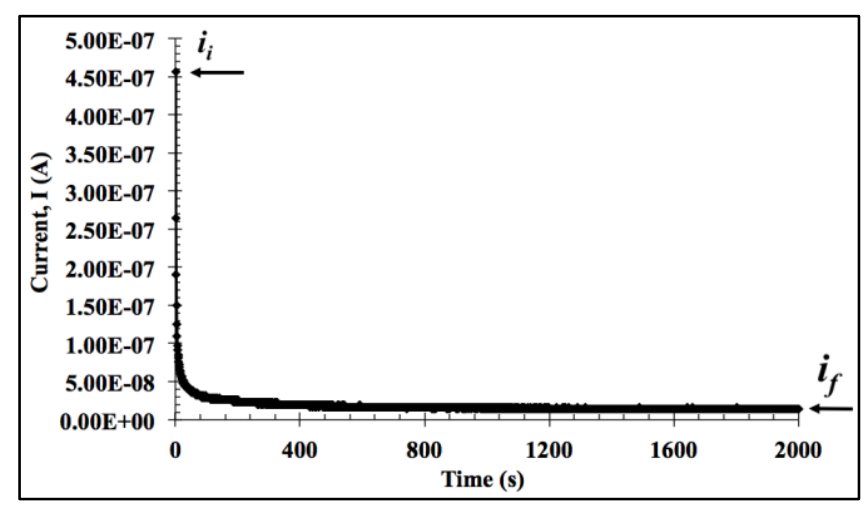

Fig. 9: Polarization current as a function of time for the highest conductivity of EMG30- $\mathrm{LiCF}_{3} \mathrm{SO}_{3}-\mathrm{EC}$.

Fig. 10 (a-e) depicts the FESEM image of pure EMG30, EMG30$35 \mathrm{wt} \% \mathrm{LiCF}_{3} \mathrm{SO}_{3}, \mathrm{EMG} 30-40 \mathrm{wt} \% \mathrm{LiCF}_{3} \mathrm{SO}_{3}, \mathrm{EMG} 30-45 \mathrm{wt} \%$ $\mathrm{LiCF}_{3} \mathrm{SO}_{3}$ and EMG30-LiCF $\mathrm{SO}_{3}$-EC system, respectively at 10000x magnification. Fig. 10 (a-c) shows the roughness of the surface structure of EMG30 was reduces with the increase of salt. It possibly arises from random distribution and dissociation of salt, which introduces the topological disorder in the polymer electrolyte system. The dissociation of salt caused the interaction between salt and the polymer host. The smooth morphology is closely related to the reduction of polymer crystallinity in the presence of salt [31]. The addition of more salt loading (45 wt.\% of $\mathrm{LiCF}_{3} \mathrm{SO}_{3}$ ) makes the surface morphology of the polymer electrolytes becomes rough with rumples (Fig. 10 (d)) This result is possibly due to ion aggregation in this sample. It elucidates the different settling rate of the matrix when more excess salt is present. In other words, the excess of lithium salts content reduces the dissolution of charge carriers. These structures are the result of neutral ion aggregate formation that forms a drop in ionic conductivity results. The addition of EC in the polymer electrolyte systems shows an improvement in surface morphology as demonstrated in Fig. 10 (e). This plasticizer successfully smoothens the surface and strongly proves the amorphous nature of the plasticized polymer electrolytes. Predictably, large volumes of plasticizer are being entrapped in the polymer matrix, thus increasing the flexibility of the segmental motion. This result ensures good electrodeelectrolyte contact, which in agreement with the conductivity results.

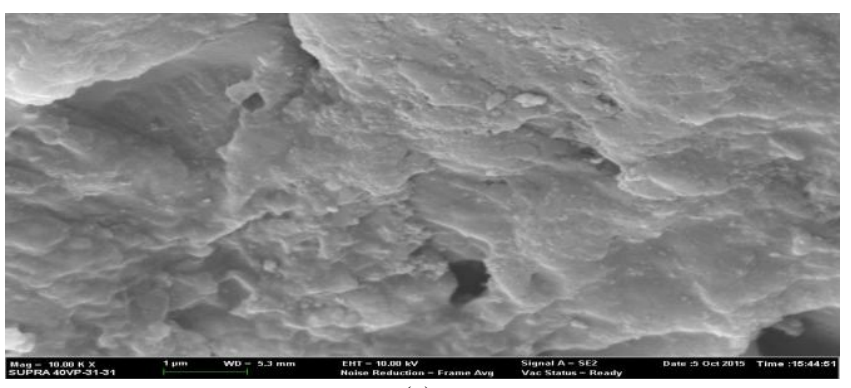

(a)

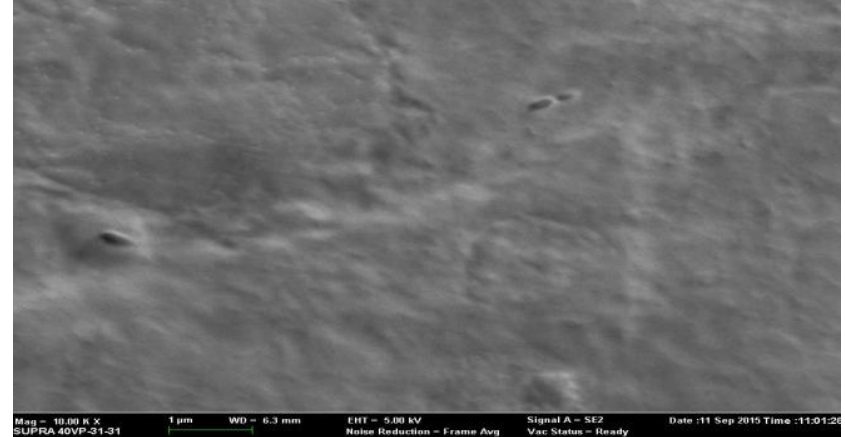

(b)

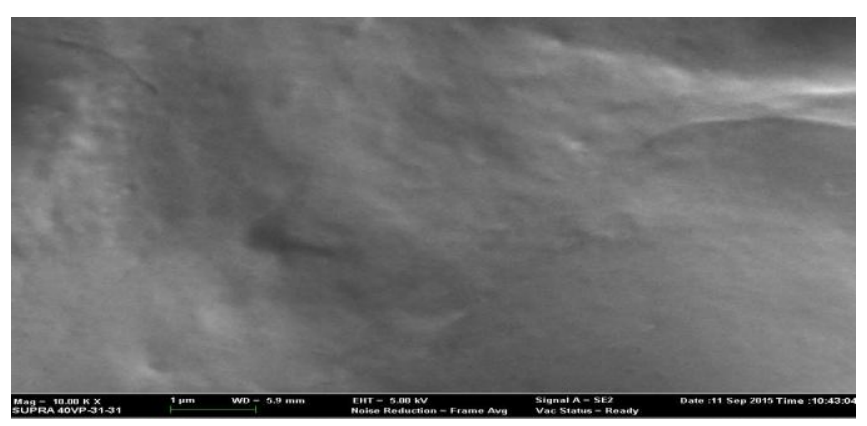

(c)

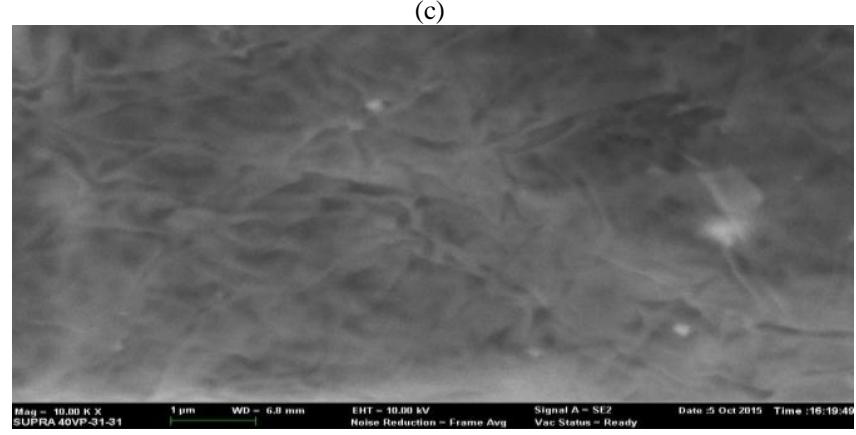

(d)

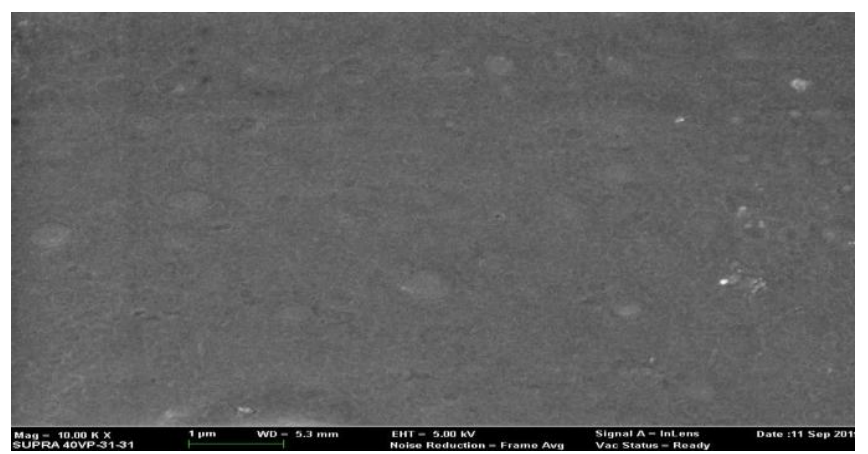

(e)

Fig. 10: FESEM micrograph of (a) EMG30 (b) EMG30-35 wt.\% $\mathrm{LiCF}_{3} \mathrm{SO}_{3}$ (c) EMG30-40 wt. $\% \mathrm{LiCF}_{3} \mathrm{SO}_{3}$ (d) EMG30-45 wt. $\% \mathrm{LiCF}_{3} \mathrm{SO}_{3}$ (e) $\mathrm{EMG} 30-\mathrm{LiCF}_{3} \mathrm{SO}_{3}$-EC.

\section{Conclusion}

Gel polymer electrolyte (GPEs) EMG30 which is doped with the $\mathrm{LiCF}_{3} \mathrm{SO}_{3}$ film was prepared by solution casting technique to give the highest conductivity $4.83 \times 10^{-3} \mathrm{~S} \mathrm{~cm}^{-1}$ at room temperature. The temperature dependence study shows that the ion migration can be explained by VTF model which the ion migration occurred in the amorphous phase. Transference number results are found 0.83 and 0.96 for EMG30- $\mathrm{LiCF}_{3} \mathrm{SO}_{3}$ and $\mathrm{EMG} 30-\mathrm{LiCF}_{3} \mathrm{SO}_{3}-\mathrm{EC}$ electrolyte systems; respectively reveal the charge transport is predominantly due to ions. Surface morphological analysis proved the sample with the highest conductivity exhibited most homogenous in nature. 


\section{Acknowledgement}

Authors would like to thank Ministry of Higher Education (MOHE) Malaysia for funding this research under the grant FRGS 5/3 (031/2017) and Institute of Sciences, Universiti Teknologi MARA (UiTM) for the facilities provided.

\section{References}

[1] S. Guhan, N. Venkatesan Prabhu, and D. Sangeetha, "Development of sulfonated poly (ether ether ketone) electrolyte membrane for applications in hydrogen sensor," Polym. Sci. Ser. A, vol. 53, no. 12 pp. 1159-1166, 2011.

[2] A. Gonzalez, E. Goikolea, J. A. Barrena, and R. Mysyk, "Review on supercapacitors: Technologies and materials," Renew. Sustain. Energy Rev., vol. 58, pp. 1189-1206, 2016.

[3] J. Reiter, O. Krejza, and M. Sedlaříková, "Electrochromic devices employing methacrylate-based polymer electrolytes," Sol. Energy Mater. Sol. Cells, vol. 93, pp. 249-255, 2009.

[4] L. Yue, J. Ma, J. Zhang, J. Zhao, S. Dong, Z. Liu, G. Cui, and L. Chen, "All-solid-state polymer electrolytes for high-performance lithium-ion batteries," Energy Storage Mater., vol. 5, pp. 139-164, 2016.

[5] S. a. M. Noor, a. Ahmad, I. a. Talib, and M. Y. a. Rahman, "Morphology, chemical interaction, and conductivity of a PEO ENR50 based on solid polymer electrolyte," Ionics (Kiel)., vol. 16, no. 2, pp. 161-170, Oct. 2009.

[6] J. Park, K. Enomoto, T. Yamashita, Y. Takagi, K. Todaka, and Y. Maekawa, "Polymerization mechanism for radiation-induced grafting of styrene into alicyclic polyimide films for preparation of polymer electrolyte membranes," J. Memb. Sci., vol. 438, pp. 1-7, 2013.

[7] E. Passaglia, M. Bertoldo, S. Coiai, S. Augier, S. Savi, and F. Ciardelli, "Grafting of methyl methacrylate onto natural rubber in supercritical carbon dioxide," Polym. Adv. Technol., vol. 19, no. April, pp. 560-568, 2008.

[8] N. K. and A. M. M. A. R. H. Y. S. A.H. Ahmad, "Effects of Plasticiser on the Lithium Ionic Conductivity of Polymer Electrolyte PVC-LiCF3SO 3," Ionics (Kiel)., vol. 11, pp. 442-445, 2005

[9] M. F. Z. Kadir, S. R. Majid, and A. K. Arof, "Plasticized chitosanPVA blend polymer electrolyte based proton battery," Electrochim. Acta, vol. 55, no. 4, pp. 1475-1482, 2010.

[10] F. Latif, S. F. Mohamad Zamri, and M. Aziz, "Anions Effect on the Electrical Properties of PMMA/ENR 50 Blend Electrolytes," Adv. Mater. Res., vol. 1107, no. JUNE, pp. 145-150, 2015

[11] M. Aziz, F. Latif, C. L. Chew, and N. Katun, "The Impedance Spectroscopy Studies of PVC / ENR 50 / LiCF 3 SO 3," Solid State Phenom., vol. 111, pp. 67-70, 2006.

[12] L. N. Sim, S. R. Majid, and A. K. Arof, "Characteristics of PEMA PVdF-HFP blend polymeric gel films incorporated with lithium triflate salt in electrochromic device," Solid State Ionics, vol. 209_ 210, pp. 15-23, 2012.

[13] P. Sharma, D. K. Kanchan, and N. Gondaliya, "Effect of ethylene carbonate concentration on structural and electrical properties of PEO-PMMA polymer blends," Ionics (Kiel)., vol. 19, no. 5, pp. 777-785, Sep. 2013.

[14] A. M. M. Ali, R. H. . Subban, H. Bahron, T. Winie, F. Latif, and M. Z. a. Yahya, "Grafted natural rubber-based polymer electrolytes: ATR-FTIR and conductivity studies," Ionics (Kiel)., vol. 14, no. 6, pp. 491-500, Jan. 2008.

[15] M. S. Su'ait, A. Ahmad, H. H. Hamzah, and M. Y. Rahman, "Preparation and characterization of PMMA-MG49-LiClO4 solid polymeric electrolyte," J. Phys. D. Appl. Phys., vol. 42, p. 5pp, Jun. 2009.

[16] K. S. Yap, L. P. Teo, L. N. Sim, S. R. Majid, and A. K. Arof, "Investigation on dielectric relaxation of PMMA-grafted natural rubber incorporated with LiCF3SO3," Phys. B Condens. Matter, vol. 407, no. 13, pp. 2421-2428, Jul. 2012

[17] A. M. M. Ali, R. H. Y. Subban, H. Bahron, M. Z. A. Yahya, and A S. Kamisan, "Investigation on modified natural rubber gel polymer electrolytes for lithium polymer battery," J. Power Sources, vol. 244, pp. 636-640, 2013

[18] A. Ahmad, H. Harun, and M. A. Yarmo, "Preparation and Characterization of $49 \%$ Poly ( Methyl Methacrylate ) Grafted Natural Rubber ( MG49 ) - Stannum ( IV ) Oxide ( SnO 2 ) -
Lithium Salt Based Composite Polymer Electrolyte," Int. J. Electrochem. Sci., vol. 7, pp. 8309-8325, 2012.

[19] S. P. Low, a. Ahmad, H. Hamzah, and M. Y. a. Rahman, "Nanocomposite solid polymeric electrolyte of $49 \%$ poly(methyl methacrylate)-grafted natural rubber-titanium dioxide-lithium tetrafluoroborate (MG49-TiO2-LiBF4)," J. Solid State Electrochem., vol. 15, no. 11-12, pp. 2611-2618, Dec. 2010.

[20] M. S. Su'ait, A. Ahmad, H. Hamzah, and M. Y. A. Rahman, "Preparation and characterization of PMMA-MG49-LiClO 4 solid polymeric electrolyte," J. Phys. D. Appl. Phys., vol. 42, no. 5, pp. 1-5, Mar. 2009.

[21] K. S. Yap, L. P. Teo, L. N. Sim, S. R. Majid, and A. K. Arof, "Plasticised polymer electrolytes based on PMMA grafted natural rubber-LiCF3SO3-PEG200,” Mater. Res. Innov., vol. 15, pp. 34-38, 2011.

[22]R. Yoksan, "Epoxidized Natural Rubber for Adhesive Applications," J. Nat. Sci., vol. 42, pp. 325-332, 2008.

[23] P. Saramolee, N. Lopattananon, and K. Sahakaro, "Preparation and some properties of modified natural rubber bearing grafted poly(methyl methacrylate) and epoxide groups," Eur. Polym. J., vol 56, pp. 1-10, 2014.

[24] K. Nazir, A. F. Aziz, N. I. Adam, M. Z. A. Yahya, and A. M. M. Ali, "Effect of epoxidation on $30 \%$ poly(methyl methacrylate)grafted natural rubber polymer electrolytes," AIP Conf. Proc., vol. 1674 , p. 20020,2015

[25] J. R. MacCallum and C. A. Vincent, Polymer Electrolytes Reviews1 (Eds). London: Elsevier, 1987.

[26] A. M. M. Ali, M. Z. A. Yahya, H. Bahron, and R. H. Y. Subban, "Electrochemical studies on polymer electrolytes based on poly(methyl methacrylate)-grafted natural rubber for lithium polymer battery," Ionics (Kiel)., vol. 12, no. 4-5, pp. 303-307, Nov. 2006.

[27] M. Kumar and S. . Sekhon, "Role of plasticizer's dielectric constant on conductivity modification of PEO-NH4F polymer electrolytes," Eur. Polym. J., vol. 38, no. 7, pp. 1297-1304, 2002.

[28] L. N. Sim, "Investigation on PEMA/PVdf-HFP Blend Polymer Electrolytes Incorporated With Carbonate -Based Plasticizers and Imidazolium-Based Ionic Liquids," Universiti of Malaya, 2012.

[29] a. M. M. Ali, M. Z. a. Yahya, H. Bahron, R. H. Y. Subban, M. K. Harun, and I. Atan, "Impedance studies on plasticized PMMA-LiX [X: CF3SO3-, N(CF3SO2)2-] polymer electrolytes," Mater. Lett., vol. 61, no. 10, pp. 2026-2029, Apr. 2007.

[30] O. Bohnke, G. Frand, M. Rezrazi, C. Rousselot, and C. Truche, "Fast ion transport in new lithium electrolytes gelled with PMMA . 1. Influence of polymer concentration," Solid State Ionics, vol. 66 , pp. 97-104, 1993.

[31] S. A. Mohd Noor, "Solid polymeric electrolyte of poly(ethylene)oxide-50\% epoxidized natural rubber-lithium triflate (PEO-ENR50-LiCF3SO3)," Nat. Sci., vol. 2, no. 3, pp. 190-196, 2010 . 